\title{
Implementación de guías de práctica clínica de la Asociación de Enfermeras de Ontario-RNAO: Una experiencia de enfermería en un hospital colombiano
}

\author{
Implementation of Clinical Practice Guidelines of the Registered \\ Nurses Association of Ontario-RNAO: A Nursing Experience in a \\ Colombian Hospital
}

\section{Implementação de diretrizes de prática clínica do Ontário Nurses Association-RNAO: Uma experiência de enfermagem um hospital colombiano}

Olga Lucía Cortés, Enf., MSc., PhD.*

Aracelly Serna-Restrepo, Enf., Esp. **

Luz Dary Salazar-Beltrán, Enf., Esp. ***

\section{Resumen}

Introducción: La implementación de guías de práctica clínica (GPC) es una estrategia que tiene como fin el aumento del uso de la evidencia en el contexto de toma de decisiones y construcción de políticas en salud. Profesionales de enfermería de la Fundación CardioinfantilInstitución de Cardiología (FCI-IC) establecieron un convenio colaborativo con la Asociación de Enfermeras de Ontario (RNAO), Canadá, con el fin de implementar y evaluar tres de sus GPC en adultos hospitalizados. Objetivo: Describir el diseño, proceso y evaluación preliminar del plan de implementación entre julio 2012 y

\author{
Yudi Andrea Rojas-Castañeda, Enf., Esp. **** \\ Silvia Cabrera-González, Enf., MSc. ***** \\ Indira Arévalo-Sandoval, Enf., Esp. ******
}

diciembre 2014. Metodología: La selección de GPC se realizó previo diagnóstico de necesidades y factibilidad: dos preventivas y una de cuidado. Las GPC de prevención fueron: Una de caídas y otra de úlceras por presión (UPP). La GPC de cuidado fue valoración y manejo de úlceras de pie diabético. Los indicadores seleccionados para las GPC prevención fueron frecuencia de evaluación y confirmación del riesgo y tasa de eventos (caídas y UPP). En la GPG cuidado se evaluó el porcentaje de pacientes valorados (miembros inferiores y pies) en admisiones; porcentaje de educación; y porcentaje de pacientes con reducción (curación) de herida en pie (del 50\%). Resultados: La evaluación del riesgo con re-valoración a las 24-72 horas (caídas/UPP) fue realizada en todos los pacientes

\footnotetext{
Enfermera, Especialista Cuidado Crítico, Maestría en Ciencias Clínicas de Cuidado, Doctora en Investigación Clínica, Investigadora Asociada al Departamento de Investigaciones, Fundación Cardioinfantil-IC, Bogotá, Colombia.

** Enfermera, Especialista Gestión Humana y Desarrollo Organizacional, Sub Directora Departamento Enfermería, Fundación Cardioinfantil- IC, Bogotá, Colombia.

*** Enfermera, Especialista Gerencia Calidad en Salud, Coordinadora Servicios Ambulatorios, Fundación Cardioinfantil- IC, Bogotá, Colombia.

**** Enfermera, Especialista Epidemiología, Coordinadora Grupo Cuidado Piel, Fundación Cardioinfantil- IC, Bogotá, Colombia.

${ }_{* * * * * * * \text { Enfermera, Magisteren Pediatría. Coordinadora Educación, Fundación Cardioinfantil-IC. Bogotá, Colombia. }}$
}

Autor de Correspondencia: Olga Lucía Cortés, Investigadora Asociada al Departamento de Investigaciones, Calle 163a \#13B-60. Torre H. Fundación Cardioinfantil-IC. Bogotá, D.C. Colombia. Correo electrónico: olgacortesf@gmail.com 
admitidos. Se observó una reducción en el uso de barandas, y una baja reducción en caídas. No se observó una reducción en UPP, pero hubo una limitación del tipo de UPP solo a grado II. Aumentó la valoración en miembros inferiores, la educación y el cuidado de úlceras en pie en pacientes diabéticos. Conclusiones: La implementación de las GPC y el trabajo en red con la RNAO han orientado nuestra práctica del cuidado en enfermería basado en la evaluación del riesgo y eventos. Limitaciones en el nivel de evidencia de algunas recomendaciones han promovido el desarrollo de una nueva investigación. [Cortés OL, SernaRestrepo A, Salazar-Beltrán LD, Rojas-Castañeda YA, Cabrera-González S, Arévalo-Sandoval I. Implementación de guías de práctica clínica de la Asociación de Enfermeras de Ontario-RNAO: Una experiencia de enfermería en un hospital colombiano. MedUNAB 2016; 19(2): 103-114].

Palabras clave: Práctica clínica basada en la evidencia; Enfermería; Guía de práctica clínica; Enfermería basada en la evidencia; Mejoramiento de la calidad.

\section{Abstract}

Introduction: The implementation or Clinical Practice Guidelines (CPG) is a strategy that whose goal is to increase the use of evidence in the context of decision making and building health policies. Nurses of The "Cardioinfantil-IC" Foundation (FCl-IC) established a collaborative agreement with the Registered Nurses Association of Ontario, Canada (RNAO), in order to implement and evaluate three of its GPC in adult inpatients. Objective: This paper describes the design, process and preliminary evaluation of an implementation plan between July 2012 and December 2014. Methodology: The selection of the CPG was made after an initial needs diagnosis and a feasibility analysis. Two CPGs were based on prevention: one about fall and another one about pressure ulcers (PU); and one more based on direct care related to assessment and management of diabetic foot ulcers. The indicators selected for CPG about prevention were frequency of evaluation and confirmation of risk and event rate (falls and UP). In the CPG about care was evaluated the percentage of assessed patients (lower limbs and feet) in admissions, the percentage of patient/family education, and the percentage of patients with foot injury reduction (50\%). Results: The evaluation of the risk for falls and pressure ulcers with a reassessment ranging from 24 to 72 hours was performed in all patients that were admitted. There was a reduction in the use of guardrails, and a low reduction in falls. There was no change in the rate of pressure ulcers but the incidence of ulcers was limited to grade II. The assessment in lower limbs, the education, and care about foot ulcers in diabetic patients increased. Conclusions: The implementation process of GPC, as a result of the networking with the RNAO, has guided nursing care practice based on the assessment of risk and events. Some limitations in the level of evidence of some recommendations have promoted

\section{Introducción}

Las guías de práctica clínica (GPC) constituyen un tipo de síntesis del más alto nivel de la literatura, basadas en la mejor evidencia existente sobre recomendaciones del cuidado (1). Dada la información avanzada que contienen, son elementos the development of a new research. [Cortés OL, SernaRestrepo A, Salazar-Beltrán $L D$, Rojas-Castañeda YA, Cabrera-González S, Arévalo-Sandoval I. Implementation of Clinical Practice Guidelines of the Registered Nurses Association of Ontario-RNAO: A Nursing Experience in a Colombian Hospital. MedUNAB 2016; 19(2): 103-114].

Keywords: Evidence-based practice; Nursing; Practice guideline; Evidence-based nursing; Quality improvement.

\section{Resumo}

Introdução: A implementação das diretrizes de prática clínica (CPG) é uma estratégia que visa aumentar o uso de provas no contexto da tomada de decisões e na construção de políticas no sistema de saúde. Os profissionais de enfermagem da Fundação Cardioinfantil-IC (FCl-IC) estabeleceram uma cordo de colaboraçãocom a Associação de Enfermeiras de Ontário (RNAO), Canadá, a fim de implementar e avaliartrês de seus GPC em adultos hospitalizados. Objetivo: Este artigo descreve o desenho, processo e avaliação preliminar do plano de implementação entre Julho de 2012 e Dezembro de 2014. Metodologia: A seleção de GPC foi realizada antes do diagnóstico das necessidades e da viabilidade: Duas preventivas e uma do cuidado. As GPC de prevenção foram: Uma das quedas e outra de úlceras por pressão (UPP). A GPC do cuidado foi a avaliação e gestão de úlceras do pé diabético. Os indicadores selecionados para as GPC de prevenção foram freqüentemente de avaliação e da confirmação do risco e a taxa de eventos (quedas e UPP). Na GPG, o cuidado foiavaliado a percentagem de pacientes avaliados (pernas e pés) nasadmissões; a percentagem de educação; e percentagem de pacientes comredução (cura) da ferida no pénum (50\%). Resultados: A avaliação de risco comumare avaliação dentro de 24-72 horas (quedas/ UPP) foi realizada a todos os pacientes internados. Foi observada uma redução na utilização de trilhos, e uma baixoredução das quedas. Nenhuma redução foi observada em UPP, mas houveuma limitação do tipo de UPP sóao tipo II. Aumentou a avaliação nas pernas, a educação e o cuidado nas úlceras no péem pacientes diabéticos. Conclusões: $A$ implementação da CPG e trabalhoem rede com o RNAO guiaramnoss aprática de cuidados de enfermagem com base na avaliação de risco e eventos. Limitaçõ esquantoao nível da evidência de algumas recomendações têm promovido o desenvolvimento de novas pesquisas. [Cortés OL, Serna-Restrepo A, SalazarBeltrán LD, Rojas-Castañeda YA, Cabrera González S, Arévalo-Sandoval I. Implantação das diretrizes de prática clínica da Association de enfermeiras de Ontario-RNAO: Uma experiência de enfermagem um hospital colombiano. MedUNAB 2016; 19 (2): 103-114].

Palavras-chave: Prática clínica baseada em evidências; enfermagem; Guia de prática clínica; Enfermagem baseada em evidências; Melhoria de qualidade.

que ayudan a la toma de decisiones de los profesionales de la salud sobre situaciones del cuidado específicas (1). Al igual que las revisiones sistemáticas, las GPC revisan, evalúan y combinan la evidencia (2). Sin embargo, las GPC van más allá de las revisiones sistemáticas por cuanto en ellas se combinan todos los aspectos y valores relevantes que determinan una decisión clínica (riesgo, pronóstico, edad, 
costos, valores del paciente, alcances de la institución entre otros) (2,3). Las GPC refinan preguntas clínicas y mantienen un equilibrio entre los beneficios y el riesgo de una recomendación. El proceso metodológico para la realización de GPC se basa en el consenso de un grupo de investigadores, expertos, clínicos de cuidado directo y pacientes, quienes examinan la evidencia cuidadosamente y deciden sobre la base de sus implicaciones (2). Las recomendaciones finales van dirigidas hacia un país, una región, un hospital o una clínica. Estas recomendaciones pueden diferir dependiendo del país o hacia el grupo o región al cual van dirigidas, como un área rural o urbana, un hospital universitario, y por estas diferencias algunos expertos prefieren no producir recomendaciones sino resúmenes de la evidencia (3-5).

Las GPC de alta calidad son producidas por numerosas organizaciones interesadas en la calidad del cuidado con el fin de crear centros de alta excelencia como es el caso de la Asociación de Enfermeras de Ontario, RNAO (3). La construcción de GPC, para la RNAO, está fundamentalmente orientada hacia el mejoramiento de los resultados de los pacientes promoviendo así la reducción de la variabilidad en el cuidado $(6,7)$. La revisión y/o modificación de las recomendaciones dependerá de la aparición de nueva evidencia en enfermería que supere la anterior (ejemplo, desarrollo de nuevos ensayos clínicos, o meta-análisis). Aunque se conoce la importancia de las GPC en el impacto en la seguridad de los individuos y la calidad en el cuidado en salud, su utilización aún no se considera una práctica usual en nuestros hospitales (8-11). Dado que las guías presentan los cuidados que producen mejores resultados, su utilización racional conduce a una variación adecuada de la práctica $(4,12)$. Las GPC proporcionan un enfoque para la educación continua, permiten el uso eficiente de los recursos, una mejor evaluación del costo beneficio del cuidado, y exponen las deficiencias de la literatura existente sugiriendo el paso hacia el desarrollo de investigaciones futuras $(11,12)$.

Con el fin de alcanzar la excelencia en el cuidado, líderes del Departamento de Enfermería de la Fundación Cardioinfantil iniciaron un proyecto conjunto con la RNAO. El propósito del proyecto colaborativo, iniciado en el 2012, fue el de implementar, evaluar y validar, sobre la base de un estudio de factibilidad y adaptabilidad previo en un centro hospitalario, tres GPC de la RNAO (13). Las GPC fueron seleccionadas sobre la base de un diagnóstico de necesidades acorde con el plan de implementación institucional de guías de práctica de enfermería (14). Estas guías fueron las siguientes: prevención de caídas y lesiones en adultos mayores; valoración y manejo de úlceras de pie en pacientes con diagnóstico de diabetes; y valoración del riesgo y prevención de úlceras por presión (15-17).

Adicionalmente, esta colaboración incluyó el compromiso con el registro de los eventos de cada guía en la base de datos de la RNAO (NQuIRE), con el fin de alimentar la base mundial de resultados post implementación de cada GPC.
Esta información de eventos a nivel mundial permitirá evaluar el impacto de las GPC de la RNAO en varios países y centros del mundo al tiempo que establecerá el nivel de avance de cada país o centro con respecto al progreso general. Por último, esta colaboración pretendió promover la divulgación de los avances y progresos resultantes de la implementación de cada GPC, con el fin de exponer la importancia del uso de GPC y los avances en términos de calidad y seguridad del cuidado en una institución de cuarto nivel de atención en Colombia.

Aunque en Colombia se han desarrollado Guías de Práctica Clínica en Enfermería, poca evidencia existe sobre los procesos de implementación y sus resultados a corto, mediano y a largo plazo. Es así como el objetivo de este documento es el de describir la experiencia en el proceso de implementación de las tres GPC. Así mismo se pretende presentar algunos alcances en la implementación acorto plazo (2 años) en términos de resultados en la práctica clínica y las implicaciones para la práctica clínica de enfermería en la FCI-IC.

\section{Base histórica para esta implementación en la Fundación Cardioinfantil - Instituto de Cardiología}

El mejoramiento continuo de la calidad en el cuidado no es solamente un propósito institucional sino uno de los lineamientos del Ministerio de Protección Social en Colombia (18). Por eso, las mejores instituciones en términos de resultados son evaluadas como centros de excelencia en el cuidado $(18,19)$. Siendo consistentes con el propósito institucional de mejorar los resultados en los pacientes, un grupo de profesionales de Enfermería de FCIIC se dio a la tarea de impulsar este convenio colaborativo y de aprendizaje con la RNAO. Las líderes de este proyecto al igual que las directivas institucionales tuvieron como lineamiento institucional el uso de la evidencia existente (universal), la transferencia del mejor conocimiento y el desarrollo de más investigación, con el fin de alcanzar la más alta calidad en la atención en salud.

Como institución en desarrollo constante y líder en el cuidado cardiovascular, la FCI- IC y sus líderes en enfermería han trabajado en la búsqueda, actualización e implementación de estrategias de cuidado basados en la mejor evidencia (20). La elaboración de protocolos, flujogramas de cuidado y de algunas denominadas "guías" de manejo y cuidado del paciente y su familia han sido producto de proyectos de investigación y actualización de la literatura de profesionales en enfermería $(20,21)$. Sin embargo, el desarrollo científico en enfermería progresó hacia el conocimiento de la Enfermería Basada en la Evidencia y en los nuevos conceptos de eficacia y eficiencia de estrategias con el fin de promover los resultados en los pacientes y familias (21). Así mismo, la idea de mejoramiento del cuidado y calidad avanzó hacia el reconocimiento de la importancia de las Guías de Práctica 
Clínica (GPC). Estas son compendios de recomendaciones evaluadas acorde con sus niveles de evidencia, generadas de forma sistemática, y obtenidas de los hallazgos de investigaciones de calidad (revisiones sistemáticas, meta análisis, ensayos clínicos, estudios de cohorte), por expertos probados en cada área (23).

Teniendo en cuenta que la producción de alta calidad de GPC en el mundo ha avanzado, la identificación y evaluación crítica de las mejores guías a nivel mundial y la implementación de estas se convierte en una intervención más avanzada que construir nuevas guías de nivel institucional. De esta forma se visualiza la importancia del convenio con la RNAO, una institución con experiencia en el desarrollo científico de GPC desde el año 1925, al servicio del Ministerio de Salud Canadiense con el fin de promover centros de alta calidad en el cuidado (24).

El convenio colaborativo entre la FCI-IC y la RNAO, el cual fue estructurado en el 2011 ha constituido un avance de aprendizaje y progreso en enfermería. El fortalecimiento del conocimiento científico de enfermería en la FCI-IC al lado de una institución con experiencia en la construcción de GPC ha permitido prosperaren el liderazgo científico en la profesión hacia la construcción y mantenimiento de la Fundación Cardioinfantil- Instituto de Cardiología (FCIIC) como centro de alta excelencia.

\section{Metodología}

\section{Selección de Guías de Práctica Clínica}

La Fundación Cardioinfantil- IC cuenta con un total de 9 servicios de hospitalización para adultos y un total de 3 servicios de hospitalización para niños, 90 camas de cuidado intensivo, de las cuales 19 son de neonatos, 20 son de pediatría y 51 son de adultos y cuenta con una capacidad total de 340 camas. Evaluaciones a lo largo de 10 años en la institución, permitieron realizar un claro diagnóstico de los problemas relacionados con la aparición de eventos como caídas, úlceras en piel y úlceras en pie en pacientes diabéticos. La evaluación interna de tipo diagnóstico observó la existencia de dificultades en el uso de instrumentos de diagnóstico para evaluación del riesgo tanto para caídas como para úlceras por presión (UPP); una dificultad en el registro en la historia clínica de los eventos (posiblemente un sub-registro), prevalencia de caídas con lesiones con uso del $100 \%$ de las barandas en cama en todos los pacientes con riesgo (desde moderado - alto), y presencia importante de prevalencia de úlceras de tercer y cuarto grado. Basados en esta evaluación se seleccionaron tres GPC acorde con las necesidades y retos de cuidado identificados. Dos guías fundamentadas en cuidado preventivo $(15,17)$, como la de prevención de las caídas y lesiones derivadas de las caídas en personas mayores y la valoración del riesgo y prevención de úlceras por presión; y una guía fundamentada en cuidado curativo, valoración y manejo de úlceras de pie en pacientes diabéticos (16).

\section{Servicios de implementación y población}

Para este primer periodo de implementación (1 julio a 31 diciembre 2012), se seleccionaron servicios con alta prevalencia de eventos en población adulta ( $\geq 18$ años). Para la implementación de las guías de prevención de caídas se seleccionaron servicios de medicina interna-cirugía general y servicios de cardiología y cirugía cardiaca. Para la implementación de la guía de prevención de UPP seleccionamos dos unidades de cuidados intensivos (unidad médica y quirúrgica). Para la implementación de la guía de valoración y manejo de úlcera en pies en pacientes con diagnóstico de diabetes se optó por incluir todos los servicios de hospitalización adultos (ocho servicios en total).

\section{Indicadores/Resultados}

Los indicadores de evaluación de la implementación para la guía de valoración del riesgo y prevención de úlceras por presión incluyeron el porcentaje de pacientes admitidos que fueron valorados de forma diagnóstica con la escala de riesgo, porcentaje de revaloración a las 24 horas, pacientes en riesgo de presentar UPP (\%), y la incidencia de UPP grado II a grado IV (número pacientes con UPP/ clientes con riesgo de UPP) en periodo de tiempo determinado (Tabla 1).

Los indicadores de evaluación de la implementación de la guía de valoración y manejo de úlceras en pie de pacientes diabéticos incluyeron el porcentaje de valoración de pacientes diabéticos con úlcera en pie en las piernas y porcentaje de valoraciones en los pies en el momento de admisión; porcentaje de educación al paciente y su familia y reducción del tamaño de la herida (úlcera), al menos del 50\% en pie. (Tabla 1).

Finalmente, los indicadores de la guía de prevención de caídas y lesiones post caídas en adultos/adultos mayores incluyeron el porcentaje de pacientes valorados de forma diagnóstica con la escala de riesgo para caídas en el momento de la admisión, evaluación diagnóstica del riesgo posterior a una caída, total de individuos que ingresaron al programa de prevención de caídas, porcentaje de uso de barandas, tasa de caídas (Número caídas/ días- paciente), y lesiones post caída (Tabla 2).

\section{Proceso de implementación y monitoría}

El proceso de planeación de la implementación se inicia una vez firmado el convenio colaborativo entre las dos instituciones a mediados del año 2012. La firma de este convenio y el entrenamiento de un grupo de líderes-base permitieron el comienzo del proceso de apropiación de las GPC de la RNAO y el inicio de los procesos relacionados con la implementación.

Este convenio le dio un carácter prioritario y formal a una relación de colaboración entre profesionales de Enfermería. Los compromisos entre las dos instituciones estuvieron dados sobre la base de la investigación del impacto de las GPC. 
Tabla 1. Indicadores seleccionados de las guías de prevención y cuidado de la piel de la RNAO.

\begin{tabular}{cl}
\hline $\begin{array}{c}\text { Valoración del riesgo y prevención de } \\
\text { úlceras por presión (UPP): Código }\end{array}$ & $\begin{array}{l}\text { Objetivo: Pacientes adultos/ adultos mayores con alto y/o } \\
\text { muy alto riesgo según escala Braden }\end{array}$ \\
\hline Ulcerprev-pr001 & $\begin{array}{l}\text { Porcentaje de pacientes nuevos ingresados con valoración } \\
\text { del riesgo de desarrollar UPP }\end{array}$ \\
\hline Ulcerprev-pro02 & $\begin{array}{l}\text { Porcentaje de pacientes con revaloración del riesgo de UPP } \\
\text { en periodo específico de tiempo }\end{array}$ \\
\hline Ulcerprev-proc03 & $\begin{array}{l}\text { Porcentaje de pacientes "en riesgo de UPP" con manejo de } \\
\text { superficie de apoyo }\end{array}$ \\
\hline Ulcermgt-out01 & Incidencia de Úlceras por Presión (UPP) \\
\hline diabético: Código & Objetivo: Pacientes diabéticos >18 años con úlcera en Pie \\
\hline Amfoot-Pro01 & $\begin{array}{l}\text { Porcentaje de pacientes }>15 \text { años con DM con úlcera de pie } \\
\text { con evidencia de valoración bilateral de Extremidades }\end{array}$ \\
\hline Amfoot-Pro02 & $\begin{array}{l}\text { Porcentaje de pacientes }>15 \text { años con DM con úlcera de pie } \\
\text { con valoración completa de la úlcera }\end{array}$ \\
\hline Amfoot-Pro03 & $\begin{array}{l}\text { Porcentaje de pacientes }>15 \text { años con DM con úlcera de pie } \\
\text { con evidencia de recibir educación ellos o sus familias }\end{array}$ \\
\hline Amfoot-Out01 & $\begin{array}{l}\text { Porcentaje de pacientes }>15 \text { años con DM con úlcera de pie } \\
\text { con al menos } 50 \% \text { de reducción de la úlcera del pie en área } \\
\text { (curación) }\end{array}$ \\
\hline $\begin{array}{l}\text { Porcentaje de pacientes }>15 \text { años con DM con úlcera de pie } \\
\text { con cierre de la úlcera de pie. }\end{array}$ \\
\hline
\end{tabular}

Fuente: Base de datos de la NQuIRE

El proceso de implementación se llevó a cabo en varias fases. La primera fase incluyó el estudio riguroso de las GPC seleccionadas. El estudio comprendió el análisis de la literatura sustentando las intervenciones y la respectiva evaluación de los niveles de evidencia (Grado I a IV). Se revisaron las recomendaciones incluidas en cada una de las GPC y se seleccionaron aquellas calificadas con mejor nivel de evidencia, más necesarias para los servicios, y que

Tabla 2. Indicadores seleccionados de la guía de prevención de caídas y lesiones derivadas en personas mayores de la RNAO.

\begin{tabular}{ll}
\hline Código & Nombre del Indicador \\
\hline Falls_pro01 & Valoración del riesgo de caídas en nuevos ingresos \\
\hline Falls_pro02 & Valoración del riesgo posterior a una caída \\
\hline Falls_pro03 & Prevención de caídas \\
\hline Falls_pro04 & Uso de contenciones (Barandas) \\
\hline Falls_out01 & Porcentaje de caídas \\
\hline Falls_out02 & Caídas que causan lesión \\
\hline
\end{tabular}

Fuente: Base de datos de la NQuIRE 
pudieran ser implementadas acorde con los recursos (materiales, personales y administrativos) de la institución (Tabla 3; Tabla 4). Se evaluaron los patrones de práctica actuales, y uso de las escalas de riesgo. Se seleccionaron los instrumentos de evaluación del riesgo de cada guía (escala Stratify para caídas; Braden para UPP; Texas para úlcera en pie en paciente diabético), y los instrumentos para seguimiento y documentación necesarios (14). Se integraron las intervenciones recomendadas con sus niveles de evidencia por la RNAO con intervenciones existentes en la institución creando diagramas de flujo de atención en pacientes de riesgo conteniendo procesos de cuidado integrales como el creado por las líderes de la guía de prevención de caídas (Figura 1).
En una segunda fase se analizaron los indicadores de cada una de las GPC y se estructuraron formatos de recolección de la información de los eventos por servicios. Se realizaron modificaciones estructurales en la historia clínica electrónica con el fin de facilitar el reporte de los eventos y planes de cuidado de enfermería que incluyeran las actividades recomendaciones de cada GPC. El registro del riesgo cobra importancia en la valoración y así mismo su reporte en la historia clínica electrónica. Además, se estructuraron sistemas de comunicación (visual o por notas) en la misma historia clínica electrónica con el fin de comunicar al personal médico y equipo interdisciplinario el riesgo de cada paciente (para caídas o para UPP) de forma transversal.

Tabla 3. Recomendaciones seleccionadas de la guía de prevención de úlceras por presión de la RNAO.

\begin{tabular}{|c|c|c|}
\hline & Recomendaciones seleccionadas & $\begin{array}{l}\text { Nivel de } \\
\text { Evidencia }\end{array}$ \\
\hline \multirow{6}{*}{ Valoración } & $\begin{array}{l}\text { Valoración del estado de la piel desde la cabeza hasta los pies y posteriormente todos los } \\
\text { días a aquellos pacientes con riesgo de deterioro de la integridad cutánea. }\end{array}$ & IV \\
\hline & $\begin{array}{l}\text { El riesgo de presentar úlceras por presión se determina combinando los criterios clínicos } \\
\text { y el uso de una herramienta fiable de valoración del riesgo (ejemplo, Escala de Riesgo de } \\
\text { Braden). }\end{array}$ & IV \\
\hline & $\begin{array}{l}\text { En caso de que los pacientes deban permanecer en cama o sentados o aquellos que se } \\
\text { sometan a intervención quirúrgica, se deberá controlar la presión, la fricción y las fuerzas } \\
\text { de cizalla en todas las posiciones. }\end{array}$ & IV \\
\hline & Todas las úlceras por presión se identifican y se clasifican según los criterios del NPUAP*. & IV \\
\hline & Valoración y manejo de las úlceras por presión en estadios del I al IV. & IV \\
\hline & $\begin{array}{l}\text { Todos los datos se documentan en el momento de la valoración inicial y la valoración } \\
\text { continua. }\end{array}$ & IV \\
\hline Planificación & $\begin{array}{l}\text { Un plan personalizado de cuidados se basa en los datos de la valoración, los factores de } \\
\text { riesgo y los objetivos para cumplir con el paciente. El plan debe incluir al paciente, su } \\
\text { familia y el personal de salud. }\end{array}$ & IV \\
\hline \multirow[t]{10}{*}{ Intervención } & Programación de cambios de posición. (por ejemplo, con uso reloj) & IV \\
\hline & $\begin{array}{l}\text { Utilizar técnica adecuada para realizar los cambios de posición (no arrastra al paciente } \\
\text { sobre las sábanas, debe realizarse con ayuda de otros profesionales }\end{array}$ & IV \\
\hline & Valore el dolor y tenga en cuenta el impacto. & IV \\
\hline & Lubricar la piel evitando realizar masaje y/o fricción sobre las prominencias óseas. & Ilb \\
\hline & $\begin{array}{l}\text { Los pacientes con riesgo de úlcera por presión no deben permanecer en colchón } \\
\text { convencional. Se utiliza un colchón antiescaras de baja presión y con espuma de alta } \\
\text { densidad }\end{array}$ & la \\
\hline & $\begin{array}{l}\text { En caso de pacientes de alto riesgo que se sometan a intervención quirúrgica, conviene } \\
\text { utilizar mecanismos de alivio de la presión. }\end{array}$ & la \\
\hline & Establecer intervenciones para pacientes con permanencia en cama o sentados. & IV \\
\hline & Proteger y favorecer la integridad de la piel. & IV \\
\hline & Proteger la piel de una humedad excesiva y de la incontinencia. & IV \\
\hline & Valoración nutricional o sospechar carencias nutricionales. & IV \\
\hline
\end{tabular}

Fuente: Base datos NQuIRE NPUAP: www.npuap.org/ 
Tabla 4. Recomendaciones seleccionadas de la guía de valoración y cuidado de úlceras en pies de pacientes diabéticos.

\begin{tabular}{|c|c|c|}
\hline & Recomendaciones seleccionadas & $\begin{array}{l}\text { Nivel de } \\
\text { evidencia }\end{array}$ \\
\hline \multirow{3}{*}{$\begin{array}{l}\text { Capacitación y } \\
\text { educación al } \\
\text { paciente }\end{array}$} & $\begin{array}{l}\text { Todos los pacientes con úlcera de pie diabético o sus cuidadores deben comprender su } \\
\text { estado, los cuidados de la úlcera y control de la diabetes. }\end{array}$ & la \\
\hline & $\begin{array}{l}\text { La educación es esencial como estrategia para que los pacientes tomen parte activa en } \\
\text { los autocuidados, la prevención y reducción de complicaciones. }\end{array}$ & IV \\
\hline & $\begin{array}{l}\text { La educación se basa en la identificación de las necesidades individuales, factores de } \\
\text { riesgo, estado de la úlcera y capacidad de cicatrización. }\end{array}$ & $\mathrm{Ib}-\mathrm{IV}$ \\
\hline Valoración & $\begin{array}{l}\text { Elaborar y documentar en la historia clínica el manejo de diabetes, la medicación, examen } \\
\text { físico }\end{array}$ & $\mathrm{Ib}-\mathrm{IV}$ \\
\hline $\begin{array}{l}\text { Estado } \\
\text { vascular }\end{array}$ & $\begin{array}{l}\text { Realizar una valoración clínica de ambas extremidades para comprobar la perfusión } \\
\text { vascular y solicitar pruebas adecuadas. }\end{array}$ & $\mathrm{lb}-\mathrm{IV}$ \\
\hline Infección & $\begin{array}{l}\text { Valorar a todos los pacientes con úlcera de pie diabético para comprobar signos de } \\
\text { infección y realizar tratamiento adecuado. }\end{array}$ & Ila \\
\hline Neuropatía & $\begin{array}{l}\text { Identificar la presencia de neuropatía periférica mediante la valoración de alteraciones } \\
\text { sensoriales, autónomas y motoras. }\end{array}$ & IIIIV \\
\hline $\begin{array}{l}\text { Deformidad del } \\
\text { pie }\end{array}$ & $\begin{array}{l}\text { Valorar la presión y deformidad del pie, el modo de andar, el calzado, derivar al paciente } \\
\text { según hallazgos. }\end{array}$ & la-IV \\
\hline \multirow{3}{*}{$\begin{array}{l}\text { Valoración de la } \\
\text { úlcera }\end{array}$} & Describir y documentar las características de la úlcera. & IV \\
\hline & Identificar la localización, longitud, anchura, y profundidad de la úlcera para clasificarla. & la-IV \\
\hline & Valorar el lecho de la úlcera, exudado, olor y piel peri lesional. & IV \\
\hline $\begin{array}{l}\text { Objetivos de } \\
\text { cuidado }\end{array}$ & Definir los objetivos en función de resultados y consultar a los expertos. & IV \\
\hline Manejo & $\begin{array}{l}\text { Identificar y optimizar los factores sistémicos, locales y extrínsecos quepuedan contribuir } \\
\text { a la cicatrización. }\end{array}$ & IV \\
\hline $\begin{array}{l}\text { Factores } \\
\text { locales }\end{array}$ & $\begin{array}{l}\text { Promover cuidados locales de la herida teniendo en cuenta el desbridamiento, el control } \\
\text { de la infección y la humedad del entorno de la herida. }\end{array}$ & IV \\
\hline $\begin{array}{l}\text { Heridas del pie } \\
\text { diabético que } \\
\text { no cicatrizan }\end{array}$ & Valorar e implementar las opciones de tratamiento para heridas no cicatrizables. & IV \\
\hline Otras terapias & Considerar el uso de terapias complementarias y/o cirugía si se retrasa la cicatrización. & la-IV \\
\hline
\end{tabular}

Fuente: Base datos NQuIRE

Con el fin de asegurar el éxito de la implementación de las GPC se seleccionaron líderes espontáneos, o líderes con capacidad crítica destacados en los servicios. No se incluyeron líderes conocidos previamente en evaluación institucional como negativos. Los líderes por servicios y por turnos (mañana, tarde y noche) fueron motivados y entrenados en el contenido de las GPC, los indicadores de evaluación y las estrategias de educación con el fin de diseminar cada guía. Para este procedimiento se tuvo en cuenta la guía de liderazgo de la RNAO y el uso de otras estrategias educativas organizacionales generales y presentadas en algunas guías (23-26).

Se elaboró material educativo con el fin de realizar intervenciones de diseminación persona a persona, en grupos pequeños y en grupos grandes. Las actividades educativas de diseminación de las guías incluyeron reuniones de lectura y análisis crítico de casos, conferencias, lecturas y sesiones de monitoreo directo con el personal. La comunicación de las guías se realizó en cada oportunidad de reunión con el personal de enfermería (profesional, auxiliar y coordinación).

Con el fin de monitorear los eventos y la adherencia en cada uno de los servicios se construyeron formatos de recolección de información relacionada con el tamizaje de pacientes en los servicios seleccionados para identificar población total admitida, población total a riesgo (para caídas o para úlceras por presión), incluyendo información de los indicadores de cada guía. Las coordinadoras de 


\section{INGRESO DE PACIENTE AL SERVICIO}

$\downarrow$

Valoración de pacientes por parte de enfermería con la aplicación del Modelo de Dorothea Orem.

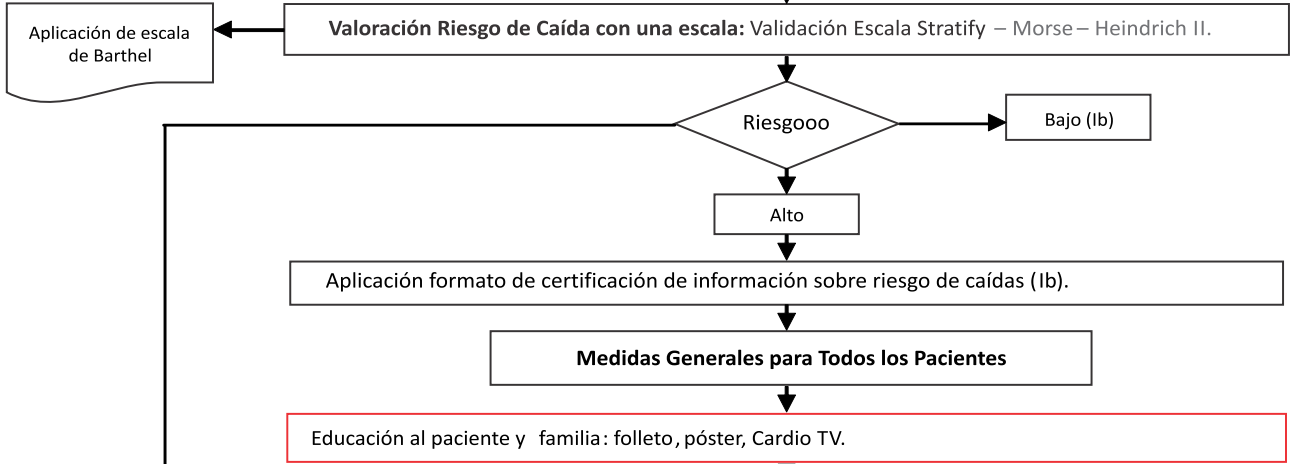

Asistencia por parte de enfermería en actividades de confort como aseo personal, eliminación, movilidad y deambulación.

Seguridad del entorno: señalización adecuada, iluminación, evitar suelos húmedos, irregulares, verificar el funcionamiento del timbre y cercanía de dispositivos de ayuda básicos parael paciente, evitar obstáculos

Utilización de elementos de seguridad como: peto perineal, canguro pediátrico, cinturón abdominal adulto y pediátrico, verificar el buen estado de cinturón de seguridad para la camilla de trasporte, y protectores de baranda cama o camilla y frenos en sillas de ruedas y camillas $(+\mathrm{lb})$

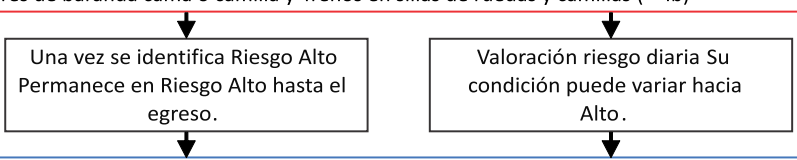

Pacientes: Identificación del paciente con manilla roja mayor de 5 años y/o punto rojo menor de 5 años (+), Cambio en la distribución de pacientes por habitación (+), hablador de identificación (+)

Formación de personal de enfermería (Lideres Champion) (+, IV)

Modificación del entorno: habitaciones, baños (pisos antideslizantes) (+)

Modificación del entorno: habitaciones, baños (piso

Modificación del personal: Relación enfermera - paciente: 1: $16(+$, la)

Relación auxiliar de enfermería - paciente 1: $8(+$, IV)

Disminución de la rotación del cuidado (Asignación plan maestro) (+, la)

Planeación del cuidado: Aplicación del Modelo de Dorothea Orem (+)

- Pacientes mayores de 80 años al ingreso, una vez al día tomar la tensión arterial acostado y sentado para identificar hipotensión ortostática e informar al médico tratante.

- Revisión de la adherencia del paciente y familia a las recomendaciones para evitar caídas: rondas clínicas de seguridad (Enf - Aux). (IV)

Revisión del tratamiento farmacológico administrado al paciente: Reducción y/ vigilancia especia con Polimedicación (4 o más medicamentos), Benzodiacepinas, Psicotrópicos, Diuréticos, Vasodilatadores... (IV)

Monitoreo de llamado: control de oportunidad al llamado para enfermería. (IV, +). Tiempo obtenido base de datos.

Reducción uso de barandas inferiores y disminución altura de camas a 60, 40, y $20 \mathrm{~cm}$. en: Torre Reinaldo Cabrera ( 64 camas), 2PHA ( 45 camas), 3PHA ( 29 camas) y 4 PHA ( 32 camas) (IV)

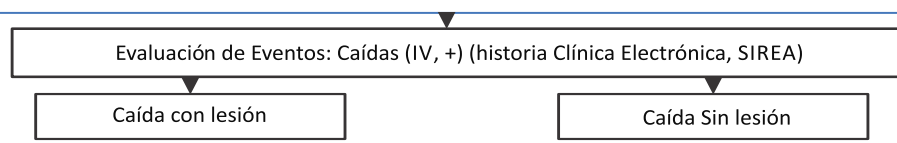

Intervenciones de la Guía de Prevención de Caídas de la RNAO* y Política y Guía para reducir el riesgo de daño causado por caídas $\mathrm{FCl}(+)$

Figura 1. Flujograma aplicación guía de prevención de caídas basada en la evidencia. 
enfermería de los turnos de la mañana, tarde y noche recolectaron inicialmente esta información con supervisión de las líderes base de la guía de prevención de caídas y prevención de úlceras por presión. Dicha información se validó y verificó en revisión de historias clínicas para ser registrada en la base de datos de la RNAO (NQuIRE) trimestralmente (19-20).

\section{Plan de análisis de resultados}

El plan de análisis de los resultados de los indicadores incluyó una revisión trimestral por año de los reportes de los resultados de los indicadores por GPC, de tal forma que para este reporte se incluyeron 8 trimestres (4 para el año 2013 y 4 para el año 2014). Los indicadores se reportaron según lo indicado por la RNAO (NQuIRE) en proporciones y/o tasa de eventos.

\section{Resultados}

\section{Aspectos generales de la implementación}

Las tres guías se implementaron en toda la institución en un periodo de 6 meses hasta diciembre de 2012. Sin embargo, solo se realizaron los reportes de resultados de los servicios acordado para tal reporte con la RNAO. Todo el personal estuvo involucrado en las sesiones tanto de presentación del proyecto colaborativo como en las sesiones educativas individuales relacionadas con cada GPC. En general se realizaron 8 sesiones de promoción institucional del proyecto colaborativo y10 sesiones tutoriales de la RNAO durante todo el proceso de implementación. Se efectuó el entrenamiento de 800 profesionales/auxiliares cuidadores en 6 sesiones en grupos grandes; y se planificaron 6 sesiones dirigidas solo a líderes de los servicios seleccionados con una dedicación de 30 horas por líder. Se organizaron equipos de trabajo para la monitoria de la adherencia de las guías, incluyendo médicos y otros profesionales de la salud distintos a enfermería.

\section{Resultados de la implementación de las GPC}

El periodo de implementación se considera entre el 1 de julio al 31 de diciembre de 2012. Se implementaron las tres guías acordadas con la RNAO con el fin de iniciar la evaluación y validación de estas en la FCI-IC para el periodo comprendido entre 1 de enero 2013 y el 31 de diciembre 2014. Los resultados se obtuvieron de los datos reportados de nuestra institución en la base de datos de la NQuIRE (http://rnao.ca/bpg/initiatives/nquire).

Para la evaluación de la guía de prevención de caídas y lesiones por caídas en adultos mayores se incluyeron 2,208 pacientes reclutados en 3 servicios de cardiología y cirugía cardiaca, y 4,154 pacientes en 3 servicios de medicina interna-cirugía. Entre los resultados más destacados de la implementación de esta guía está la adherencia del personal de enfermería representada en el uso de la escala de riesgo Stratify en el 100\% de los pacientes nuevos admitidos; y en la evaluación del riesgo del $100 \%$ de pacientes posterior a una caída. La tasa de caídas se redujo de 15.0 a 12.0 por 1,000 días/paciente admitidos. Se observó además una reducción en el uso de las barandas alrededor del $80 \%$ en pacientes a riesgo por el personal de enfermería (Figura 2 y Figura 3).

Para la evaluación de la guía de valoración y prevención de úlceras por presión se incluyeron dos unidades de cuidado intensivo (Una unidad de cuidado médico y otra unidad de cuidado quirúrgico) con un total de 5,952 pacientes admitidos. Los resultados reportaron una evaluación del $100 \%$ de los pacientes en el momento de la admisión con escala de Braden, con re-evaluación a las 24 horas (adherencia). El 100\% de los pacientes tuvo oportunidad de protección con colchón anti-escaras, como política institucional. La tasa de úlceras por presión osciló entre 2.04.0 por 1,000 clientes admitidos, con una incidencia de úlceras clasificadas de grado II (Figura 4). No se presentaron úlceras grado III y IV.

Finalmente, la guía de valoración y manejo de úlceras en pie diabético, aunque incluyó el reclutamiento de pacientes en todos los servicios de adultos ( 8 en total), contó para esta evaluación (denominador) con 87 pacientes con diagnóstico de diabetes y úlcera en uno o en ambos pies. Los resultados evidenciaron un incremento del $100 \%$ en actividades de cuidado a pacientes diabéticos como las valoraciones en miembros inferiores (Figura 5), valoración de pies y úlceras (Escala de Texas) (Figura 6) y aumento en la educación impartida al paciente y su familia con reporte en historia clínica completa (Figura 7). La evaluación de la reducción del tamaño en la úlcera en pie fue del $30 \%$ (Figura 8).

\section{Discusión}

El proceso de implementación, aunque requirió de varias fuentes para su desarrollo, se puede resumir en varias fases. En primera instancia se basó en la identificación de problemas o necesidades de cambio que vendrían a ser superadas por la implementación de estas tres GPC de la RNAO. Pero, es importante tener en cuenta que las GPC no se pueden implementar sin antes realizar un proceso de análisis, discusión y estudio de factibilidad, que permitan su utilización total o parcial, adaptada a la realidad de una institución. Este proceso además incluyó un estudio de las posibles barreras al tiempo que incluyó el análisis de las recomendaciones de la GPC, la posibilidad de adaptación de las recomendaciones y la implementación del cambio en la institución.

El cambio en la institución es una constante y específicamente la implementación de GPC es de vital importancia en los resultados clínicos de la institución. A nivel general los resultados más importantes logrados hasta 


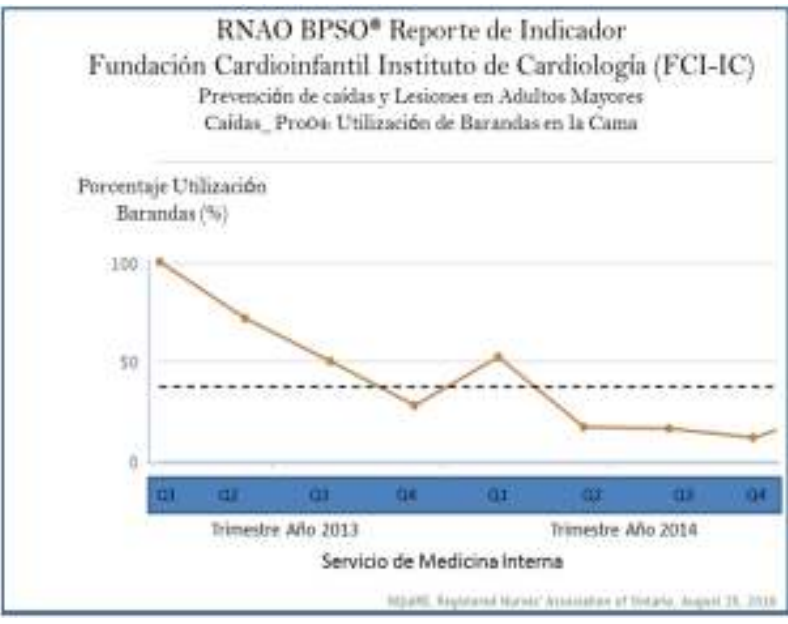

Figura 2. Tasa de utilización de barandas en la cama en servicio de medicina interna y cirugía.

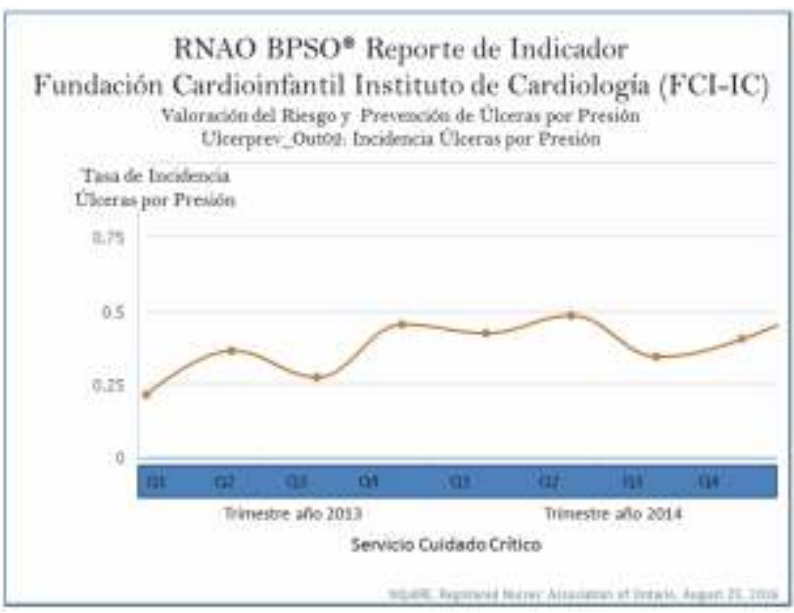

Figura 4. Tasa de incidencia de úlceras por presión Grado II.

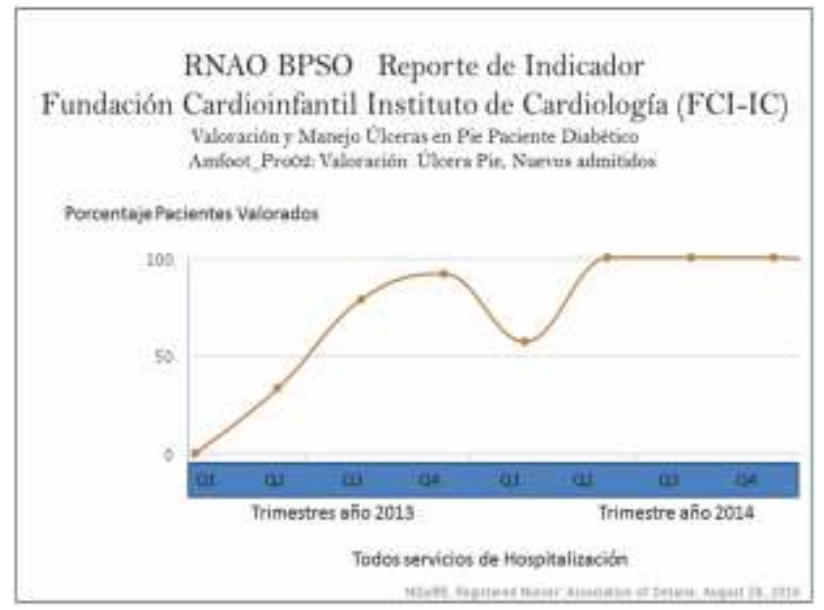

Figura 6. Aumento en la evaluación de pies con úlceras y registro en historia clínica.

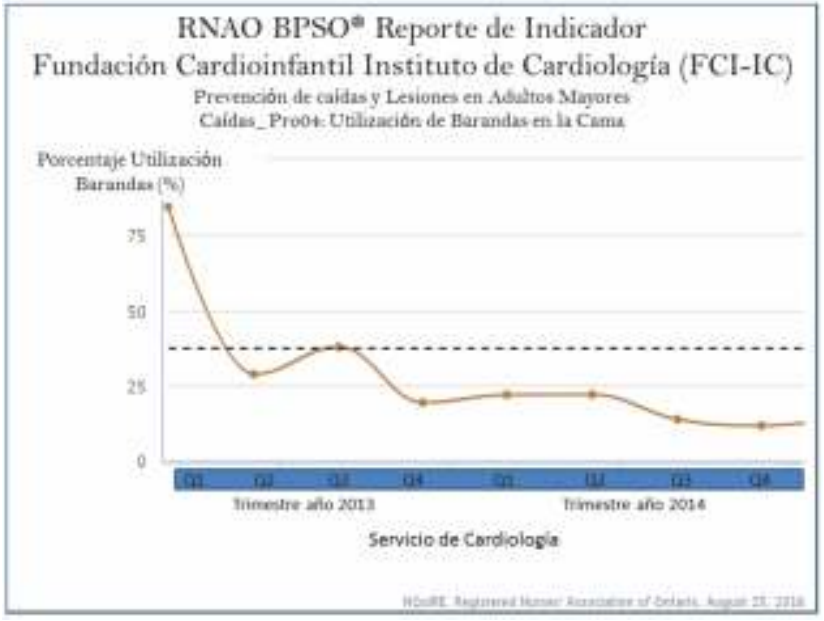

Figura 3. Tasa de utilización de barandas en la cama en servicio de cardiología.

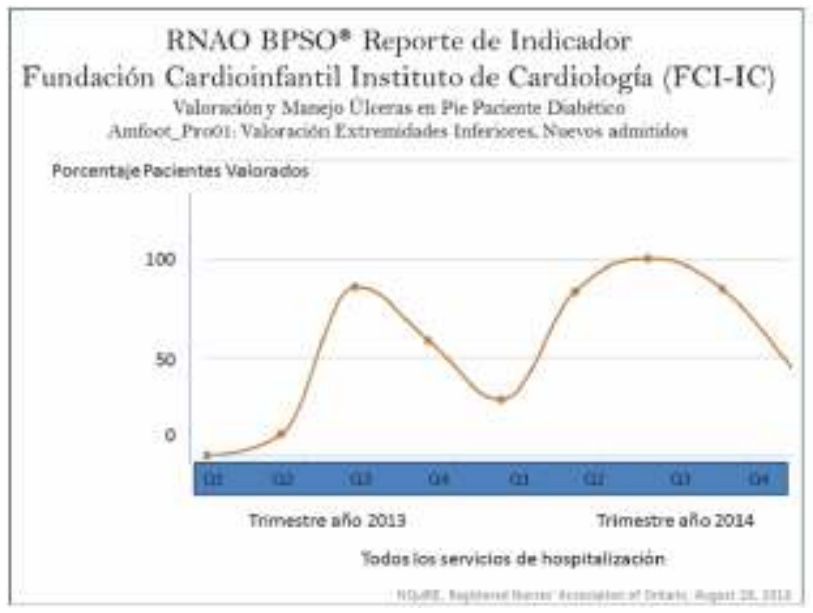

Figura 5. Aumento en la valoración de extremidades inferiores y registro en historia clínica.

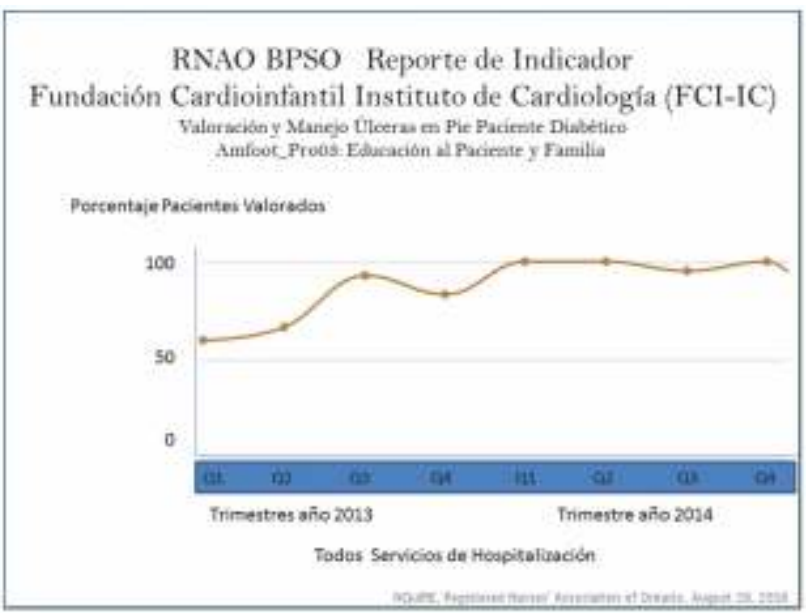

Figura 7. Incremento en la educación a pacientes diabéticos con úlcera en pie. 


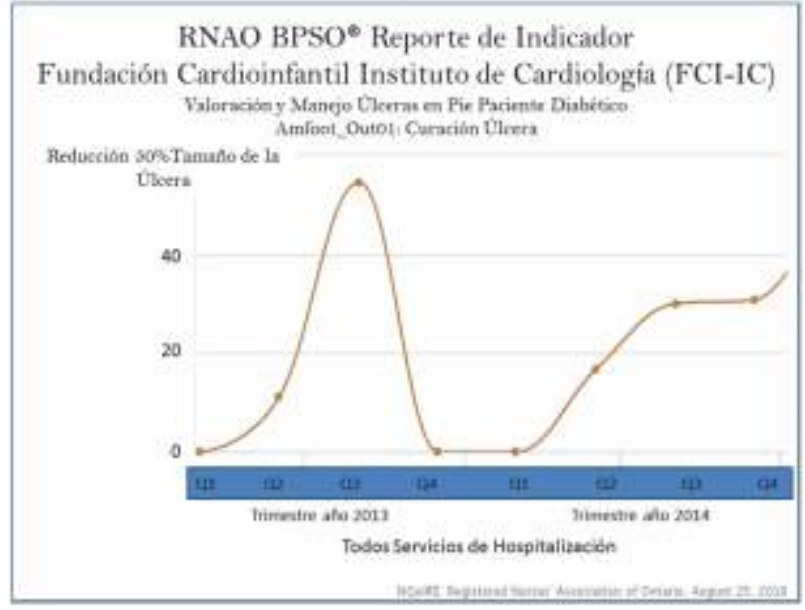

Figura 8. Porcentaje de reducción (cierre herida) en el tamaño de la úlcera en pie de paciente diabético.

la fecha (corto plazo, 2 años post implementación) de reporte de este estudio se relacionaron con la importancia que el personal de enfermería y de salud proporcionó al uso de las escalas de riesgo (y actualmente los está realizando). El personal de enfermería utilizó al 100\% las escalas de evaluación del riesgo de caídas (Stratify) y prevención de UPP (Braden) en el momento de admisión y posterior a las 24 horas. Aunque las escalas se utilizaron al 100\%, y dado que esto no asegura la validez del puntaje y la varianza entre enfermeras, el grupo de prevención de UPP y de caídas realizaron de forma quincenal educación de soporte dirigida y/o en grupo, una vez se identificaron problemas de validez en los puntajes. En general se observó una leve reducción en la tasa de caídas, que ha sido superada en los reportes del 2016. En relación a la tasa de UPP en este periodo post implementación de las guías se mantuvo en un nivel similar al que tenían las unidades de cuidado crítico antes de iniciar la implementación. Es explicable dadas las características de los pacientes hospitalizados en nuestro centro con una alteración moderada y/o severa del gasto cardiaco, y con alteración en la perfusión tisular. Los resultados identificados con la Guía de Cuidado de la úlcera en pie en pacientes diabéticos muestran un aumento en la valoración de miembros inferiores y una valoración formal de la úlcera con reporte completo en la historia clínica. Este resultado es contundente dado que la evaluación de esta información en la historia clínica pre implementación tuvo resultados entre bajos y aceptables. Aunque ha cobrado gran importancia esta evaluación de pacientes diabéticos con úlcera en pie, algunos problemas relacionados con el sistema de salud como la imposibilidad de continuidad en el cuidado de úlceras y la amputación de partes del pie o de la extremidad han limitado los alcances del cuidado por el personal de Enfermería.

En general la implementación de las GPC y la conexión continua con la red de grupos y centros de la RNAO ha permitido vislumbrar la importancia de este proyecto colaborativo en términos de aprendizaje y cuidado dirigido teniendo en cuenta el riesgo de cada individuo. El análisis trimestral de las estadísticas (eventos) relacionadas en cada servicio ha mejorado el entendimiento del valor del reporte de eventos y el impacto en el cuidado en relación a los resultados. El entusiasmo general y la apropiación individual de este conocimiento han contribuido a la formación de equipos de implementación y la identificación y formación de líderes institucionales.

Este proceso de implementación institucional ha conducido hacia un mejoramiento en la documentación de los eventos, evaluación y análisis crítico de los indicadores en grupos y por servicios, mejorando el impacto en las evaluaciones nacionales e internacionales en términos de acreditación. El trabajo en red con otros centros a nivel mundial ha permitido comprender los avances y progresos como institución y los alcances comparados con otros centros de niveles nacional y mundial.

Las limitaciones de este proceso pueden haber afectado los resultados. Los análisis de la NQuIRE no tienen en cuenta aspectos como número de enfermeras por paciente, tipo de complicaciones del paciente, que en la FCI-IC están relacionados con problemas cardiovasculares y de bajo gasto cardiaco, edad del paciente, número de intervenciones y reintervenciones quirúrgicas, estado de conciencia y número de medicamentos que recibe un paciente, o el aumento en el reporte de eventos comparado con el periodo de pre implementación, entre otros.

En cuanto a la sostenibilidad de la implementación se ha planeado como parte de la transferencia del conocimiento. La Transferencia de cada GPC se viene planeando desde la inducción y entrenamiento que se realiza a los nuevos profesionales de enfermería y auxiliares; y mediante la continuidad de las intervenciones a partir de la valoración integral del paciente, la planeación, ejecución y evaluación del cuidado. Se espera entregar un informe al finalizar el año 2016.

La identificación de recomendaciones con limitaciones en sus niveles de evidencia ha promovido la planeación y desarrollo de varios estudios de investigación en enfermería y la promoción de la prescripción de intervenciones de enfermería en el grupo de piel. La creación de la cultura del cuidado en relación al riesgo es uno de los aspectos más valiosos de esta implementación.

\section{Referencias}

1. Dearholt S, Dang D. John Hopkins Nursing EvidenceBased Practice: Model and Guidelines. 2a ed. USA: Sigma Theta Tau International; 2012. Disponible en: http://samples.sainsburysebooks.co.uk/9781935476788 sample_187851.pdf

2. DiCenso A, Virani T, Bajnok I, Borycki E, Davies B, et al. A toolkit to facilitate the implementation of clinical practice guidelines in healthcare settings. Hosp Q. 2002 Spring; 5(3):55-60.

3. Bajnok I, Grinspun D, Lloyd M \&McConnel H. Liderando mejoras de calidad mediante el desarrollo, 
implementación, y medición de las guías de buenas prácticas de Enfermería. MedUNAB 2015; 17(3):155-162.

4. Albornos-Muñoz L, González-María E, Moreno-Casbas T. Implantación de guías de buenas prácticas en España. Programa de centros comprometidos con la excelencia de cuidados. MedUNAB 2015;17(3):163-169.

5. Silva-G A. Implementación de guías de buenas prácticas clónicas elaboradas por Registered Nurses Association of Ontario en curriculum de Enfermería Universidad de Chile. MedUNAB 2015; 17(3):182-189.

6. Grinspun D, Lloyd M, Xiao S, Bajnok I. Midiendo la calidad del cuidado de Enfermería basado en la evidencia: NQuIRE-Sistema de Indicadores de la Calidad en Enfermería para reportar y evaluar. MedUNAB 2015; 17(3): 170-175.

7. Registered Nurses' Association of Ontario [Internet]. Canadá: [Citado 20 agosto 2016]. Best Practice Guidelines. [1 pantalla]. Disponible en:http://www.rnao.org/bestpractices/index.asp.

8. Agency for Healthcare Research and Quality [Internet]. USA: [Content last review November 2014; acceso 20 de agosto de 2016]. Clinical Guidelines and Recommendations. [1pantalla]. Disponible en:http://www. ahrq.gov/-clinic/epcix.htm Evidence Based Practice.

9. Ouimet M, Landry R,Amara N,Belkhodja O. What factors induce health care decision-makers to use clinical guidelines? Evidence from provincial health ministries, regional health authorities and hospitals in Canada. SocSci Med.2006 Feb;62(4):964-76.

10. 10.Colón-Emeric CS, Lekan D, Utley-Smith $Q$, Ammarell N, Bailey D, et al. Barriers to and Facilitators of Clinical Practice Guideline Use in Nursing Homes. -J Am Geriatr Soc. 2007 Sep; 55(9): 14049.

11. Dobbins M, Davies B, Danseco E, Edwards N, Virani T: Changing nursing practice: Evaluating the usefulness of a best-practice guideline implementation toolkit. Can J Nurs Res. 2005; 18(1): 34-45.

12. Goode CJ, Tanaka DJ, Krugman M, O'Connor PA, Bailey C. Outcomes from use of an evidence-based practice guideline. Nurs Econ. 2000, 18(4): 202-7.

13. Fundación Cardio-Infantil IC. Informe anual de gestión. Fundación Cardio-Infantil IC. Bogotá, 2009-2012. [ S I i d e S h a re ]. D i s p o n i ble e n : http://www.slideshare.net/pasante/1caso-de-xitofundacin-cardioinfantil-turismo-de-salud.

14. Registered Nurses' Association of Ontario. Herramienta de implantación de buenas prácticas. Guía de Buenas Prácticas. 2nd ed. Toronto, Canadá: Registered Nurses Association of Ontario. 2012. Disponible en: http://rnao.ca/sites/rnao-ca/files/Toolkit_en15.pdf.

15. Registred Nurses' Association of Ontario. Prevention of falls and fall injuries in the older adult. (Revised). Toronto, Canadá: Registered Nurses' Association of Ontario. 2011. Disponible en: http://rnao.ca/bpg/ guidelines/prevention-falls-and-fall-injuries-older-adult.

16. Registered Nurses' Association of Ontario. Assessment and management of foot ulcers for people with diabetes.2nd ed. Toronto, ON: Registered Nurses' Association of Ontario. 2013. Disponible en: http:// rnao.ca/bpg/guidelines/assessment-and-managementfoot-ulcers-people-diabetes-second-edition.

17. Registered Nurses' Association of Ontario. 'Risk assessment \& prevention of pressure ulcers. (Revised). Toronto, Canada: Registered Nurses Association of Ontario. 2011. Disponible en: http://rnao.ca/bpg/ guidelines/ risk-assessment-and-prevention-pressure-ulcers.
18. Ministerio de Salud y Protección Social. Manual de implementación de guías de práctica clínica basadas en evidencia, en instituciones prestadoras de servicios de salud en Colombia. Bogotá, Colombia: Ministerio de Salud y Protección Social. 2014. Disponible en: gpc.minsalud.gov.co.

19. Ministerio de la Protección Social. Guía metodológica para la elaboración de guías de práctica clínica con evaluación económica en el Sistema General de Seguridad Social en Salud Colombiano. Bogotá, Colombia: Ministerio de la Protección Social. 2010. Disponible en: gpc.minsalud.gov.co.

20. MarrinerA, Raile M. Modelos y teorías de Enfermería. $6^{a}$ ed. España: Editorial Elseiver; 2007.

21. Grinspun D, Virani T, Bajnok I. Nursing best practice guidelines: The RNAO (Registered Nurses Association of Ontario) Project. Hosp Q. 2001-2002; 5 (2): 56-60.

22. Edwards N, Davies B,Ploeg J,Dobbins M,Skelly J, et al. Evaluating best practice guidelines. Can Nurse.2005 Feb;101(2):18-23.

23. Registered Nurses'Association of Ontario. Developing and sustaining leadership. Best practice Guideline. International affairs \& best practice guideline. 2nd ed. Toronto, ON: Registered Nurses' Association of Ontario. 2013. Disponible en: http://rnao.ca/sites/rnaoca/files/LeadershipBPG_Booklet_Web_1.pdf.Register ed Nurses'Association of Ontario. Segunda edición, 2013.

24. Registered Nurses'Association of Ontario. Educator's Resource: Integration of best practice guidelines. 2nd ed. Toronto, Canadá: Registered Nurses'Association of Ontario. 2005. Disponible en: http://rnao.ca/bpg/ guidelines/resources/educators-resource-integrationbest-practice-guidelines.

25. Ploeg J, Skelly J, Rowan M, Edwards N, Davies B, et al. The Role of Nursing Best Practice Champions in Diffusing Practice Guidelines: A Mixed Methods Study. Worldviews Evid BasedNurs. 2010 Dec; 7 (4):238-251.

26. Registered Nurses'Association of Ontario. Assessment and Management of Pressure Injuries for the Inter professional Team. 3nd ed. Toronto, ON: Registered Nurses' Association of Ontario. 2016. Disponibleen: http://rnao.ca/bpg/guidelines/pressure-injuries. 